\title{
Kinetics of Gallbladder Emptying During Cholecystokinin Cholescintigraphy as an Indicator of In Vivo Hormonal Sensitivity
}

\author{
Laurence J. Miller ${ }^{1,2}$, Kaleeckal G. Harikumar ${ }^{1,2}$, Aditya J. Desai ${ }^{1}$, Hassan Siddiki ${ }^{2}$, and Ba D. Nguyen ${ }^{3}$ \\ ${ }^{1}$ Department of Molecular Pharmacology and Experimental Therapeutics, Mayo Clinic, Scottsdale, Arizona; ${ }^{2}$ Division of \\ Gastroenterology and Hepatology, Department of Internal Medicine, Mayo Clinic, Scottsdale, Arizona; and ${ }^{3}$ Division of Nuclear \\ Medicine, Department of Diagnostic Radiology, Mayo Clinic, Scottsdale, Arizona
}

\begin{abstract}
Cholecystokinin cholescintigraphy is used clinically to quantify gallbladder ejection fraction as an indicator of functional gallbladder disorder. It can also provide the opportunity to quantify an individual's responsiveness to the physiologic stimulant of gallbladder contraction, cholecystokinin, which is a major regulator of appetite and postprandial satiety. Methods: In the current work, we use cholecystokinin cholescintigraphy to quantify the kinetics of gallbladder emptying, including average and peak rates, in response to a standard cholecystokinin infusion. Results: We demonstrated that patients with no gallstones or biliary obstruction who empty their gallbladders completely in response to cholecystokinin, having an ejection fraction greater than $80 \%$, exhibit a broad range of sensitivity to this hormone. Three distinct kinetic profiles were observed, with those most sensitive to cholecystokinin achieving the earliest peak and the fastest rate of gallbladder emptying, whereas those least sensitive to cholecystokinin have the latest peak and the slowest rate of emptying. Conclusion: Patients can have abnormal cholecystokinin stimulusactivity coupling as an effect of endogenous negative allosteric modulation by membrane cholesterol. This was predicted in ex vivo studies but has not, to our knowledge, previously been demonstrated in vivo. This type of kinetic analysis provides a tool to quantify cholecystokinin responsiveness in patients and identify patients who might benefit from a drug that would positively modulate cholecystokinin action to improve their appetite regulation and to better control their weight.
\end{abstract}

Key Words: cholecystokinin; gallbladder; cholecystokinin cholescintigraphy; kinetics of gallbladder emptying; human gallbladder emptying

J Nucl Med Technol 2020; 48:40-45

DOI: $10.2967 /$ jnmt.119.233486

$\mathbf{H}$ ormone binding to and activation of cellular receptors are critical for optimal regulation of physiologic events. We

Received Jul. 10, 2019; revision accepted Aug. 12, 2019.

For correspondence or reprints contact: Laurence J. Miller, Mayo Clinic, 13400 E. Shea Blvd., Scottsdale, AZ 85259.

E-mail: miller@mayo.edu

Published online Oct. 11, 2019.

COPYRIGHT (C) 2020 by the Society of Nuclear Medicine and Molecular Imaging. now recognize that endogenous allosteric regulators are present in vivo that are capable of modulating several of these events and, thereby, of differentially adjusting the sensitivity of biologic systems in different individuals $(I)$. This provides a strong rationale for the current trend toward individualization of therapeutics.

One example of such a system is the action of the gastrointestinal peptide hormone, cholecystokinin, on the type 1 cholecystokinin receptor (CCK1R) present on vagal afferent neurons, a process important for the stimulation of postprandial satiety and appetite control (2). The sensitivity of this signaling system was recently described to vary across the population, with this sensitivity dependent on an individual's metabolic phenotype, based on an ex vivo assay of hormonal responsiveness (3). That assay required the in vitro adenoviral delivery of a wild-type CCK1R construct to the patient's buffy coat cells, where full cholecystokinin dose-response curves for stimulating intracellular calcium responses were able to quantify hormonal sensitivity (3). Elevated cellular cholesterol was found to be associated with reduced cholecystokinin sensitivity, a manifestation of abnormal stimulus-activity coupling with increased natural agonist ligand binding affinity yet reduced agoniststimulated signaling (3). However, standard clinical, biochemical, and morphometric data were unable to predict the hormonal responsiveness of a given individual (3). Responses to cholecystokinin were reduced in groups of subjects who were obese or had diabetes, particularly if the latter was poorly controlled (3). The relevance of this finding is clear, since these individuals are strong candidates for a drug that might enhance the action of cholecystokinin as a satiety agent, and abnormal stimulus-activity coupling in this regulatory system might make this type of drug ineffective in such individuals (2). Indeed, aberrant cholecystokinin stimulus-activity coupling in this cohort could explain the previous failures of cholecystokinin agonists in clinical feeding trials in obese subjects (2).

In the current project, we attempted to explore whether a broad spectrum of responsiveness to cholecystokinin would 
also be observed in vivo at natural levels of normal receptor expression in a physiologic system. For this exploration, we used a well-established clinical test, cholecystokinin provocation in hepatobiliary iminodiacetic acid scintigraphy, also called cholecystokinin cholescintigraphy (4). Although the degree of gallbladder contraction (ejection fraction) is the endpoint quantified in this standard clinical test as a determinant of gallbladder health (4), this test is also capable of providing kinetic data during the intravenous infusion of a standard dose of cholecystokinin $(0.02 \mu \mathrm{g}$ of sincalide per kilogram of body mass over $60 \mathrm{~min}$ ), although the current analytic software has not been designed for this type of analysis. By examining the anonymized kinetic data from these studies in a nonselected series of sequential clinical patients, we have confirmed the existence in vivo of the predicted broad spectrum of responsiveness to this hormone and demonstrated that this variation in sensitivity to cholecystokinin exists independent of gallbladder ejection fraction.

To be certain that observed variations in the kinetics of responsiveness did not reflect intrinsic gallbladder disease or mechanical obstruction to quantified bile flow, we also analyzed data from a subset of subjects who exhibited essentially complete gallbladder emptying in response to cholecystokinin. Although the normal value for gallbladder ejection fraction used clinically for this test is at least $38 \%$, we chose a much higher cutoff for this group-an ejection fraction of at least $80 \%$ without biliary obstruction or gallstones - to study cholecystokinin responsiveness in a select population. Analysis of the kinetics of gallbladder emptying in this select group of patients suggested the presence of 3 distinct subgroups of cholecystokinin responsiveness-each exhibiting differing temporal profiles of gallbladder emptying in response to cholecystokinin infusions, with differences in average and peak kinetic responses. Those patients with early gallbladderemptying responses to hormone also exhibited the highest peak responses and the highest average responses per unit time of all 3 groups. Those with slower responses to the cholecystokinin infusion exhibited reduced peak and average responses per unit time as well. Although there was a trend for reduced cholecystokinin-stimulated gallbladder ejection fractions and rates of gallbladder emptying associated with increased body mass, this trend did not reach statistical significance in the overall population. This finding had also been observed in the previous in vitro study, in which cholecystokinin sensitivity was felt to be one of several potential contributors to nutritional homeostasis (3). Having an assay to examine cholecystokinin responsiveness in vivo could facilitate the selection of subjects for drug trials designed to correct an aberrant servomechanism for appetite control present in a subset of the population.

\section{MATERIALS AND METHODS}

\section{Study Participants}

The Mayo Clinic Institutional Review Board approved this retrospective study of anonymized clinical data, and the need for informed consent was waived. All subjects had undergone cholecystokinin-provocation hepatobiliary iminodiacetic acid scintigraphy (cholecystokinin cholescintigraphy) as a clinically indicated investigation performed at Mayo Clinic Arizona between November 2012 and November 2016. In the standard clinical protocol, all patients were studied in the fasting state, and the dose of cholecystokinin administered was based on body weight, with all patients receiving $0.02 \mu \mathrm{g}$ of cholecystokinin-8 (8 amino acids) per kilogram intravenously by pump over $60 \mathrm{~min}$. All studies in which the raw primary scintigraphic images were available for kinetic analysis were included in the initial review. Subjects with gallstones, those in whom the gallbladder did not promptly fill with radioactivity, and those in whom the radiotracer did not enter the duodenum were excluded. Clinical records of the 238 included subjects were reviewed to extract potentially relevant metrics; however, because this study was retrospective, such data were often incomplete. Nevertheless, the scintigraphic data of every subject in the study were completely reanalyzed by a single investigator who was masked to all the other clinical metrics.

\section{Cholecystokinin Cholescintigraphy Data Analysis}

All digital data from clinical studies were ported to the Phillips workstation, where regions of interest for the gallbladder and for subtraction of background over the liver were chosen. The software then displayed the profile of specific signal over the gallbladder over time during the study. The time point just before initiation of gallbladder contraction was chosen as the zero point, and the point of maximal contraction was also noted. This allowed more consistent data handling, since it was learned that the nuclear medicine technologists who had originally handled the clinical testing had not uniformly identified the time point when intravenous infusion of the standard dose of cholecystokinin was initiated. The signal over the gallbladder was quantified at the zero time point, $6 \mathrm{~min}, 10 \mathrm{~min}, 20 \mathrm{~min}$, and the time point when gallbladder emptying was most complete. This provided measurements of overall ejection fraction (percentage of $100 \%$ of the specific gallbladder signal) and those fractions emptied over the first $6 \mathrm{~min}, 6-10 \mathrm{~min}, 10-20 \mathrm{~min}$, and 0-20 min. Rates of emptying per unit time were calculated for $0-6,6-10,10-20$, and $0-$ $20 \mathrm{~min}$, as well as overall from initiation to the point of maximal emptying. Profiles of emptying were determined by calculating changes in rates of emptying over time.

\section{Statistical Analysis}

Study group characteristics were analyzed using the MannWhitney test to compare mean values. A $P$ value of 0.05 or less was considered to be statistically significant.

\section{RESULTS \\ Study Population Characteristics}

The study population included 238 patients who satisfied the entry criteria: 158 (66.4\%) women and $80(33.6 \%)$ men (Table 1). The female predominance is not surprising, in view of the prevalence of functional biliary tract disease such as biliary dyskinesia in women (5). In the entire cohort, 
there was a broad, bimodal distribution of gallbladder ejection fractions (Fig. 1) with peaks at both ends of the spectrum, representing those with poor function and those with essentially complete gallbladder emptying. Thirty-one patients (13.0\% of the cohort) were categorized by the established clinical criterion (ejection fraction $<38 \%$ ) as having abnormal emptying. In the subgroup of patients exhibiting at least an $80 \%$ gallbladder ejection fraction, who were the focus of the kinetic studies, there were 141 patients $(59.2 \%$ of the cohort): $91(64.5 \%)$ women and 50 (35.4\%) men. The study population also exhibited broad distributions of kinetic values reflecting average rate of gallbladder emptying over the complete interval (over whatever period was necessary to achieve maximal contraction), the peak rate of gallbladder emptying, and the average rate of gallbladder emptying over the first 20 min of cholecystokinin administration (Fig. 2).

The ranges in weight and body mass index in the entire cohort were quite broad, with $77.4 \pm 20.8 \mathrm{~kg}$ and $27.1 \pm$ 6.0 being the means \pm SDs, respectively (Table 1 ). In the subgroup with essentially complete gallbladder emptying ( $\geq 80 \%$ gallbladder ejection fraction), these values were $76.8 \pm 19.9 \mathrm{~kg}$ and $26.9 \pm 6.0$, respectively-not significantly different from the entire group (Table 1). The other relevant morphometric, clinical, and biochemical parameters are also listed in Table 1.

The key kinetic parameters were also broadly distributed. Figure 2 illustrates the individual points reflecting overall gallbladder ejection fraction versus average rate of gallbladder emptying over the complete interval, peak rate of gallbladder emptying, and average rate of gallbladder emptying over the first $20 \mathrm{~min}$. The distribution of these 3 kinetic parameters in patients with gallbladder ejection fractions of $80 \%$ or higher demonstrates that even in patients with complete gallbladder emptying and no gallstones or biliary obstruction, there are broad ranges of cholecystokinin responsiveness. These data exhibit differences relative to one another, with the distribution of average rates of gallbladder contraction over the duration of this event being skewed toward lower rates, the distribution of peak rates of gallbladder emptying being more normally distributed, and the distribution of average rates of gallbladder emptying over the first 20 min of contraction being skewed toward higher rates.

Supplemental Table 1 (supplemental materials are available at http://jnmt.snmjournals.org) examines possible relationships between the 3 kinetic parameters (average rate of gallbladder emptying over the complete interval, peak rate of gallbladder emptying, and average rate of gallbladder emptying over the first $20 \mathrm{~min}$ ) relative to the clinical, biochemical, and morphometric parameters of the subjects in the group exhibiting complete gallbladder emptying. We compared the upper quintile with the lower quintile of each of the kinetic parameters of this group, as well as stratifying for sex. Unfortunately, the power of this analysis was low, since the numbers in these groups were quite small. Therefore, the differences and even the trends did not provide clear insights.

Table 2 examines possible relationships to body mass index, using an approach similar to Supplemental Table 1, comparing upper and lower quintiles of body mass index in the group of subjects exhibiting complete gallbladder emptying. The trends here were more prominent and were consistent with those to be expected. For the most obese subjects, serum triglycerides were higher, high-density lipoprotein cholesterol was lower, and blood pressure was higher.

Figure 3 focuses on the kinetic parameters for the subset of subjects with $80 \%-100 \%$ ejection fractions. There were 3 distinct kinetic profiles based on when gallbladder emptying peaked - 0-6 min (profile 1, $n=30$ ), 6-10 min (profile 2, $n=82$ ), or 10-20 min (profile 3, $n=29$ )—despite the fact that all these subjects exhibited near-complete or fully complete gallbladder emptying. Those with profile 1 also exhibited the highest average rate of gallbladder emptying $(P<0.015)$, the highest peak rate of emptying $(P<0.026)$, and the highest rate of emptying over the first $20 \mathrm{~min}(0-20 \mathrm{~min})(P<$ 0.0001). Those with profile 3 also had the slowest peak rate of emptying $(P<0.0001)$ and the slowest average rate of emptying $(P<0.0001)$.

\section{DISCUSSION}

Cholecystokinin cholescintigraphy is a common clinical test used to explore whether abdominal pain may reflect biliary dysfunction, particularly in patients with normal biliary
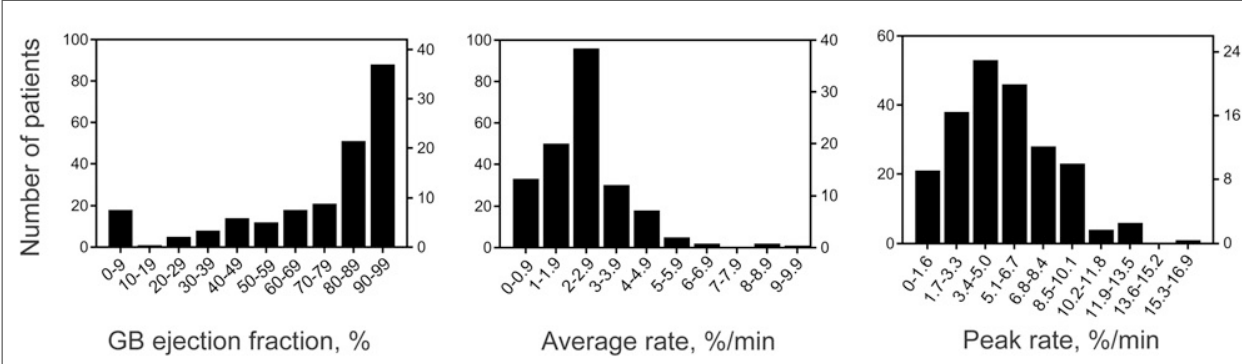

Peak rate, \%/min

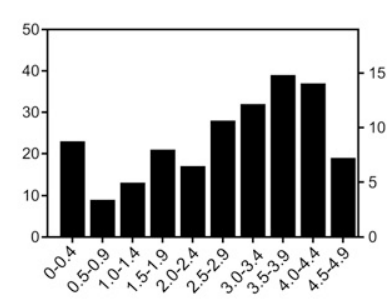

Average rate $(0-20 \mathrm{~min}), \% / \mathrm{min}$

FIGURE 1. Distribution of ejection fraction and kinetic parameters of cholecystokinin-stimulated gallbladder emptying in entire patient population. Bars reflect numbers of subjects in each bin, representing sequential $10 \%$ of total gallbladder emptying. Shown are conventional measure of gallbladder ejection fraction and key kinetic parameters of average rate over complete period of gallbladder emptying, peak rate of gallbladder emptying achieved, and average rate of gallbladder emptying over first 20 min of cholecystokinin infusion. GB = gallbladder. 


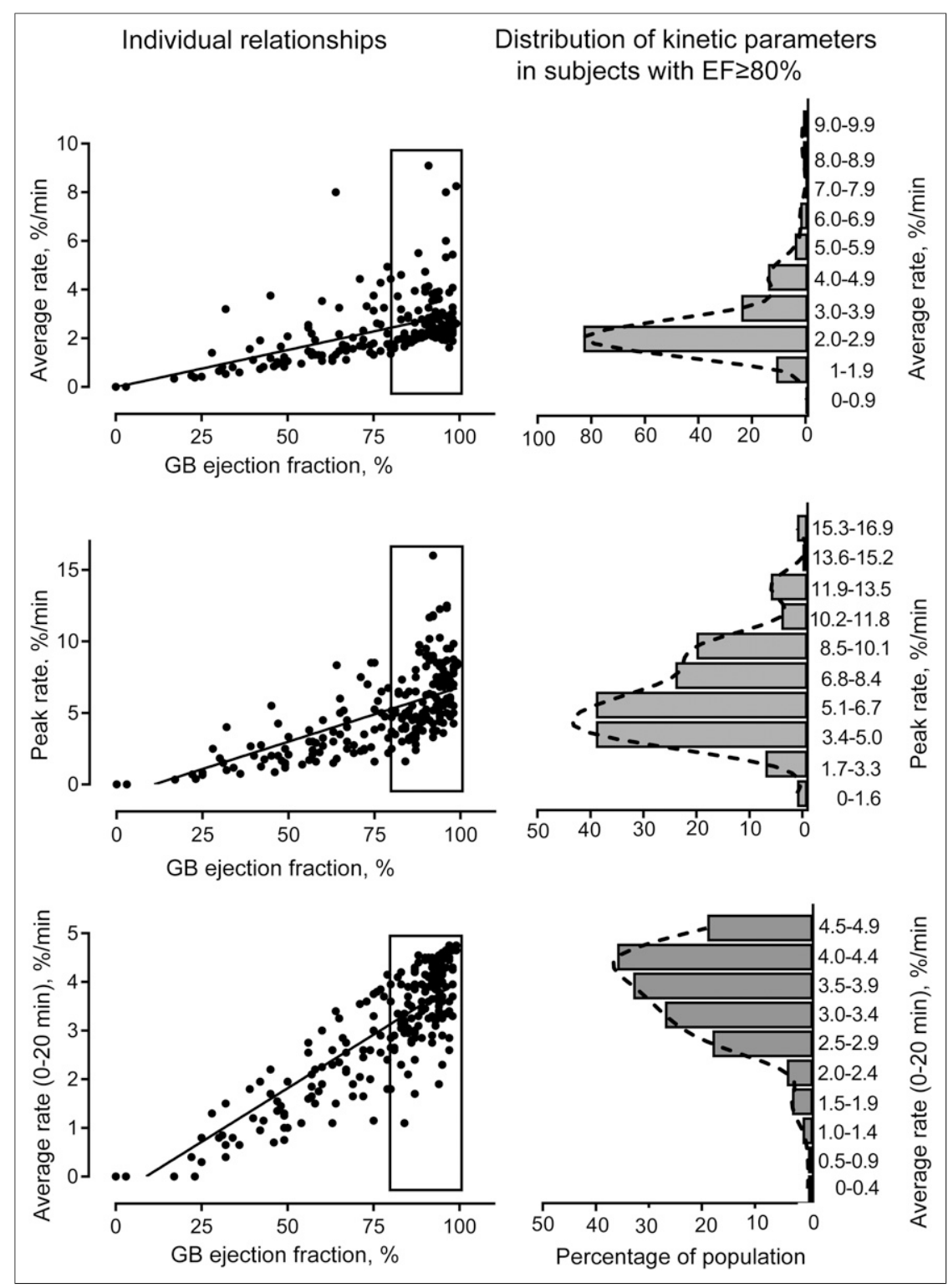

FIGURE 2. Relationships between gallbladder ejection fraction and kinetic parameters of cholecystokinin-stimulated gallbladder emptying in individuals. Shown are distributions of points representing individual data on left, and distributions of kinetic parameters in patients having gallbladder ejection fractions of at least $80 \%$. In this select group with no demonstrable gallbladder or biliary pathology, there are wide distributions of kinetics, reflecting differences in cholecystokinin responsiveness. EF = ejection fraction; $\mathrm{GB}=$ gallbladder.

ultrasonography results and no biliary calculi. Previous reviews of the use of this technique have suggested standardized procedures, including dosage and rate of hormonal (sincalide) administration over a 60 -min period (4). The data have supported the focus on gallbladder ejection fraction, with values lower than $38 \%$ identified as abnormal (4). Patients with these very abnormal levels of gallbladder emptying frequently satisfy the Rome III criteria for the diagnosis of functional gallbladder disorder and are often considered for cholecystectomy (6). Unfortunately, the pathophysiology underlying functional gallbladder disorder is not known. There are a series of other diagnoses that have been attributed to these patients, including biliary dyskinesia and sphincter-of-Oddi dysfunction, which may represent subsets of functional gallbladder disorder but are similarly poorly defined.

For the current study, we attempted to work with patients whose gallbladder function was as normal as possible, including those with complete or near-complete (80\%-100\%) gallbladder emptying in response to the infusion of the standard test dose of hormone. We were also careful to be certain that these subjects had no gallstones, had prompt uptake of the radiotracer into the liver and prompt gallbladder filling, and exhibited clear flow of the tracer into the duodenum. These selection criteria ensured the integrity of the bioassay system, with no physical factors influencing the quantified kinetics of gallbladder responsiveness. We purposefully did not include patients whose gallbladder ejection fraction was above the clinical cutoff for abnormality but was less complete than that in the included patients, because we were concerned that other influences might have been contributing to the apparent reduced hormonal responsiveness.

Like the previous ex vivo analysis of cellular calcium responses to cholecystokinin stimulation of buffy coat cells transfected to express wild-type CCK1R (3), there was a broad spectrum of hormonal responsiveness in this group of patients. The previous analysis (3) established that endogenous allosteric modulation by cellular cholesterol was one factor capable of reducing cholecystokinin responsiveness in some patients. This lipid has been shown in numerous in vitro studies $(7-10)$ to negatively affect cholecystokinin stimulus-activity coupling. Three profiles of gallbladder emptying were identified on the basis of the time necessary to achieve peak gallbladder contraction. In the group with the most rapid responses $(0-$ $6 \mathrm{~min}$ ), the rates of gallbladder ejection of radiotracer were the highest, suggesting that this group had the highest sensitivity to cholecystokinin. In the group with the slowest responses (peaking in the 10-20 min period), the rates of gallbladder ejection of radiotracer were the lowest; presumably, this group included the individuals who were least responsive to cholecystokinin. 

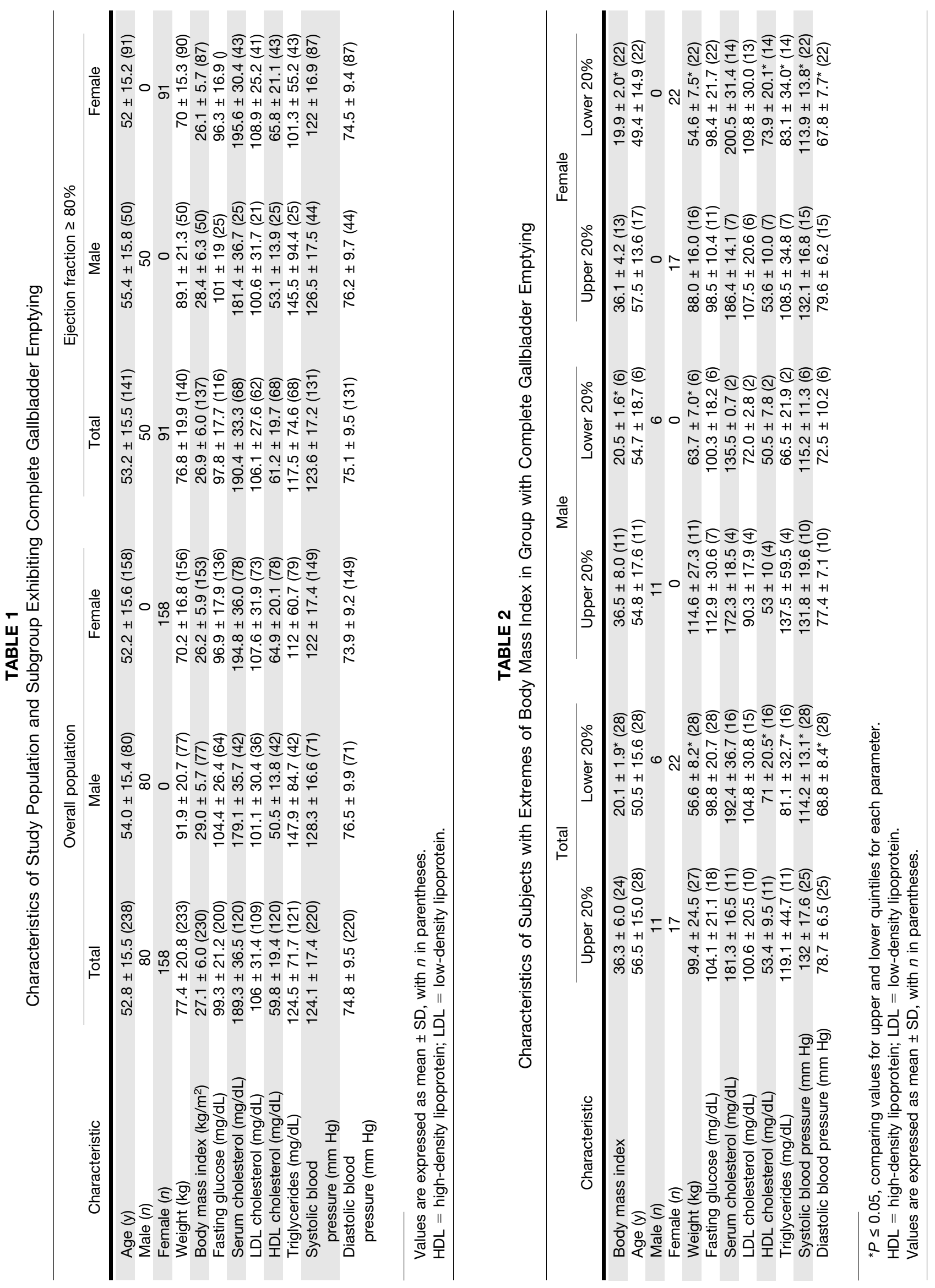

44 Journal of Nuclear Medicine Technology • Vol. 48 • No. 1 • March 2020 


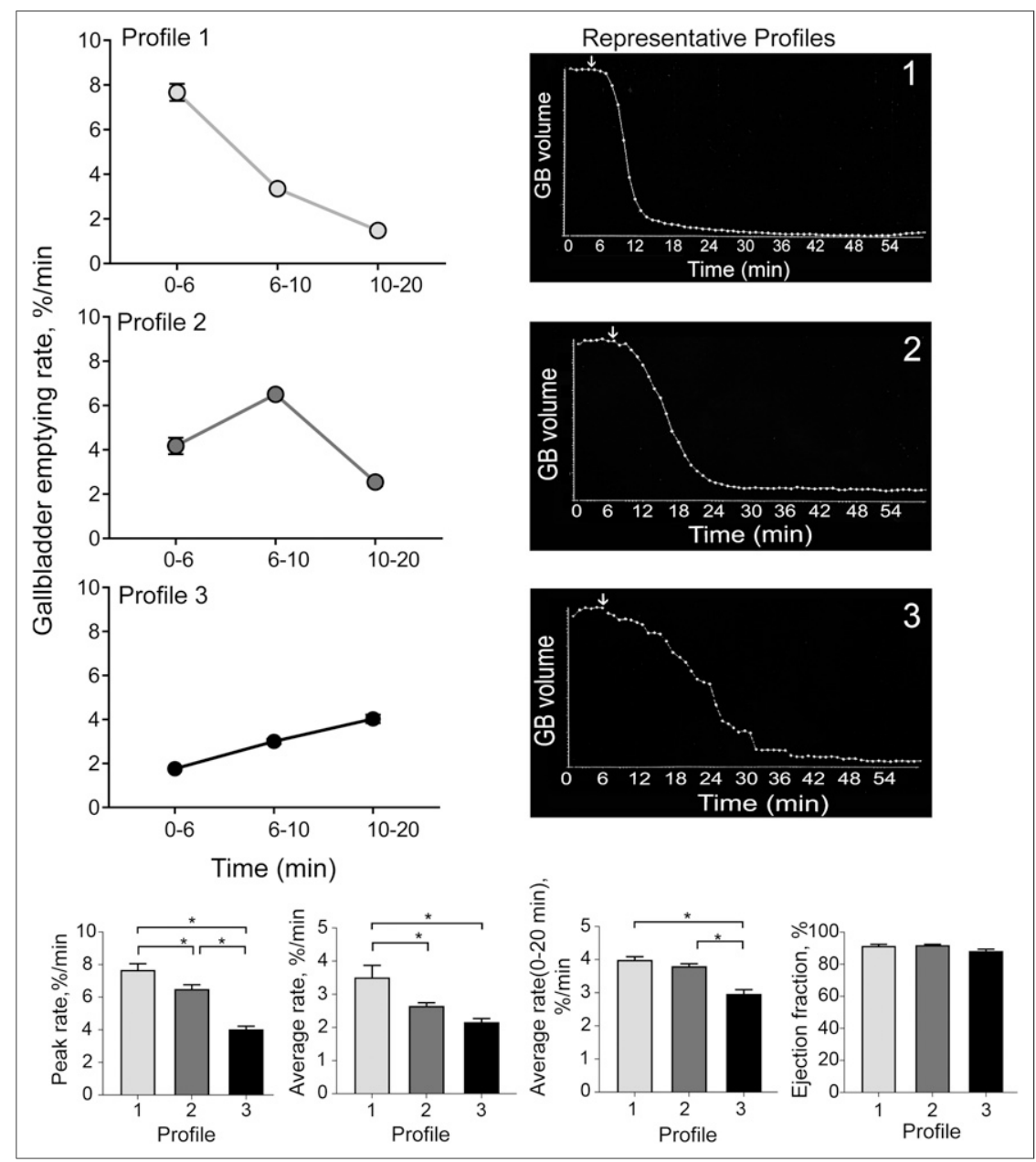

FIGURE 3. Kinetic profiles observed in patients with cholecystokinin-stimulated gallbladder ejection fractions of at least $80 \%$. Profile 1, with earliest peak response, exhibited highest rate of emptying and highest average rate of emptying. Profile 3 , with latest peak response, exhibited lowest peak rate and lowest average rate of emptying. Representative profiles are shown. GB = gallbladder.

These data provide substantial reassurance that what had been observed in the in vitro model could reflect the true in vivo responsiveness of cells that normally express CCK1R. The model studies have identified cellular cholesterol as a key determinant of responsiveness to cholecystokinin (710). However, cellular cholesterol does not necessarily correlate with serum cholesterol, and there is no existing standard clinical test that can be used to predict cholecystokinin responsiveness (3). The kinetic response to hormone observed during cholecystokinin cholescintigraphy might provide a way to quantify responsiveness to cholecystokinin that can be useful for stratification of subjects in future clinical trials. A positive allosteric modulator of cholecystokinin action could be beneficial in patients exhibiting reduced hormonal responsiveness.

\section{CONCLUSION}

Patients without gallstones or biliary obstruction who demonstrate complete gallbladder emptying in response to cholecystokinin infusion during cholescintigraphy are now shown to exhibit a broad spectrum of kinetic responses. We believe this finding reflects variation in hormonal sensitivity among individuals and could have important implications for therapies for obesity. Similar variations in cholecystokinin responsiveness were previously demonstrated in ex vivo studies, in which wildtype CCK1R was expressed into patients' buffy coat cells and dose-response curves were obtained. Such variations in vivo have not previously been reported and may reflect endogenous allosteric modulation of this receptor by membrane lipids.

\section{DISCLOSURE}

This work was supported by the Mayo Clinic. No other potential conflict of interest relevant to this article was reported.

\section{ACKNOWLEDGMENTS}

We thank Megan M. Frankart, Stephanie J. Krause, and Joseph P. Dammann for help in analyzing the hepatobiliary iminodiacetic acid scintigraphy data, and we thank Hardeep Singh, MD, for reviewing some of the clinical charts.

\section{REFERENCES}

1. van der Westhuizen ET, Valant C, Sexton PM, Christopoulos A. Endogenous allosteric modulators of G protein-coupled receptors. J Pharmacol Exp Ther. 2015;353:246-260.

2. Desai AJ, Miller LJ. Changes in the plasma membrane in metabolic disease: impact of the membrane environment on $\mathrm{G}$ protein-coupled receptor structure and function. Br J Pharmacol. 2018;175:4009-4025.

3. Desai AJ, Dong M, Langlais BT, Dueck AC, Miller LJ. Cholecystokinin responsiveness varies across the population dependent on metabolic phenotype. Am J Clin Nutr. 2017;106:447-456.

4. DiBaise JK, Richmond BK, Ziessman HA, et al. Cholecystokinin-cholescintigraphy in adults: consensus recommendations of an interdisciplinary panel. Clin Nucl Med. 2012;37:63-70.

5. Hansel SL, DiBaise JK. Functional gallbladder disorder: gallbladder dyskinesia. Gastroenterol Clin North Am. 2010;39:369-379.

6. Drossman DA, Dumitrqascu DL. Rome III: new standard for functional gastrointestinal disorders. J Gastrointest Liver Dis. 2006;130:1498-1509.

7. Xiao ZL, Chen Q, Amaral J, Biancani P, Behar J. Defect of receptor-G protein coupling in human gallbladder with cholesterol stones. Am J Gastrointest Liver Physiol. 2000;278:G251-G258.

8. Xiao ZL, Chen Q, Amaral J, Biancani P, Jensen RT, Behar J. CCK receptor dysfunction in muscle membranes from human gallbladders with cholesterol stones. Am J Physiol. 1999;276:G1401-G1407.

9. Yu P, Chen Q, Harnett KM, Amaral J, Biancani P, Behar J. Direct G protein activation reverses impaired CCK signaling in human gallbladders with cholesterol stones. Am J Physiol. 1995;269:G659-G665.

10. Behar J, Mawe GM, Carey MC. Roles of cholesterol and bile salts in the pathogenesis of gallbladder hypomotility and inflammation: cholecystitis is not caused by cystic duct obstruction. Neurogastroenterol Motil. 2013;25:283290. 